\title{
Mycobacterium abscessus-associated vertebral osteomyelitis in an immunocompetent patient: a rare case report and literature review
}

\author{
Muhammad Z. Moral $\mathbb{D}^{1} \cdot$ Khusboo Desai $^{1} \cdot$ Abdul R. Arain $^{1} \cdot$ Robert E. O'Leary ${ }^{1} \cdot$ Stefanos F. Haddad ${ }^{1}$. \\ James P. Lawrence ${ }^{1}$
}

Received: 8 January 2019 / Revised: 16 April 2019 / Accepted: 21 April 2019

(c) The Author(s) 2019. This article is published with open access

\begin{abstract}
Introduction Vertebral osteomyelitis (VO) is an uncommon infection with Staphylococcus aureus as the most commonly implicated organism. VO caused by nontuberculous mycobacteria (NTM) such as Mycobacterium abscessus (M. abscesscus) is exceedingly rare with only eight cases reported in literature.

Case presentation We report a rare case of an 82-year-old male with a remote history of trauma who was diagnosed with NTM vertebral osteomyelitis. The patient initially underwent a vertebroplasty of T12 and kyphoplasty of L1 for pathologic compression fractures. Subsequent cultures revealed M. abscessus. The patient further underwent an anterior T12-L2 corpectomy and debridement with instrumented fusion, as well as a posterior T9-L4 instrumentation and fusion. He received multi-agent antibiotic therapy; however, was ultimately unable to tolerate the aggressive treatment regimen and his prolonged postoperative course.

Discussion Nontuberculous mycobacteria vertebral osteomyelitis is exceedingly rare. NTM vertebral osteomyelitis is challenging to treat. Surgical management plays a limited role in early VO, but is the mainstay treatment in chronic VO. Early recognition of the condition and shared patient management with multidisciplinary teams is key to successfully treating cases of NTM VO.
\end{abstract}

\section{Introduction}

Since the first case was described in 1929 by Wilensky et al., vertebral osteomyelitis has remained relatively rare. A delay in diagnosis and treatment is associated with significant morbidity and mortality [1-3]. Although $S$. aureus is the most commonly isolated pathogen, other atypical organisms include Gram-negative Bacilli, Streptococci, as well as Mycobacterium species [4-8]. Treatment of vertebral osteomyelitis (VO) can be challenging, and includes both antibiotic treatment and surgical management [9]. Nontuberculous mycobacteria (NTM), particularly Mycobacterium abscessus ( $M$. abscessus), is an extremely rare cause of vertebral osteomyelitis. M. abscessus is a rapidly growing mycobacterium found in soil, plants, and aqueous

Muhammad Z. Moral

mo.z.moral@gmail.com

1 Division of Orthopedic Surgery, Albany Medical College, Albany, NY, USA environments, including municipal drinking water and sewage systems [10-12]. It has been implicated as a cause of post-traumatic infections of the skin, soft tissue, and long bones. It has also been associated with chronic lung infections, endocarditis, keratitis, and disseminated disease in immunocompromised hosts [11]. To our knowledge, only eight cases of VO caused by M. abscessus have been reported in the literature, and four were in immunocompromised hosts $[12,13]$. The treatment of NTM is difficult as it is not susceptible to most antituberculous drugs and, often requires a multi-agent drug therapy, including clarithromycin $[10,12]$. We report a rare case of VO caused by M. abscessus in an immunocompetent individual residing in a non-mycobacterium endemic region.

\section{Case presentation}

An 82-year-old male presented with a remote history of prior falls and a recent traumatic compression fracture of his L1 vertebral body. He reported no previous fractures, weight loss or antecedent pain. He was initially treated with 
a brace and underwent physical therapy. At follow-up, he had no neurological deficits but demonstrated severe recurrence of pain with progressive functional impairment refractory to conservative management. An MRI showed a worsening L1 vertebral body collapse, a T12 inferior endplate fracture, and a L2 superior endplate fracture (Fig. 1). The imaging was suspicious for a pathologic etiology. With his intractable pain and collapse at the thoracolumbar junction, we proceeded with a kyphoplasty of L1, vertebroplasty of T12, and obtained a bone biopsy for pathology and culture (Fig. 2). The initial smear testing for acid-fast bacilli (AFB) was negative with an interim negative report at 2 weeks, and final identification of $M$. abscessus at 6 weeks. The infectious disease (ID) service was consulted for further management. The smear was presumed to be a contaminant given his history and presentation.

At the 2-month follow-up, he remained neurologically intact, but continued to have functionally limiting pain. A repeat MRI demonstrated a psoas and paravertebral abscess with evidence of continued vertebral collapse. An imageguided bone biopsy confirmed the presence of $M$. abscessus and Propionibacterium acnes. Per the recommendations of the ID-team, he was started on Rocephin, then transitioned to a multi-agent regimen, including tigecycline, amikacin, meropenem, and oral-linezolid. After a completed course of antibiotics, he still continued to demonstrate clinical signs of infection with persistent pain. Again, advanced imaging confirmed progression of disease. He underwent an anterior T12-L2 corpectomy and debridement with instrumented fusion, as well as a posterior T9-L4 instrumentation and fusion (Fig. 3). Repeat AFB culture and smear redemonstrated M. abscessus. Postoperatively, he was placed on a regimen of amikacin, azithromycin, tigecycline, and meropenem.

His postoperative course was complicated by gastroparesis leading to poor oral intake and malnutrition requiring the total parental nutrition. In addition, he developed a right upper extremity deep venous thrombosis by his prior PICC line site. He required inpatient rehabilitation for functional retraining. The side effects of his multi-agent therapy included persistent nausea, antibiotic-induced Clostridium difficile colitis, and new-onset Barrett's esophagus most
Fig. 1 Sagittal (a) and axial (b) T2-weighted MRI with arrow pointing at an L1 vertebral body compression fracture concerning for infection or neoplasm. Markedly abnormal signal within the L1 vertebral body, with significant involvement of $\mathrm{T} 12$, and the superior aspect of L2
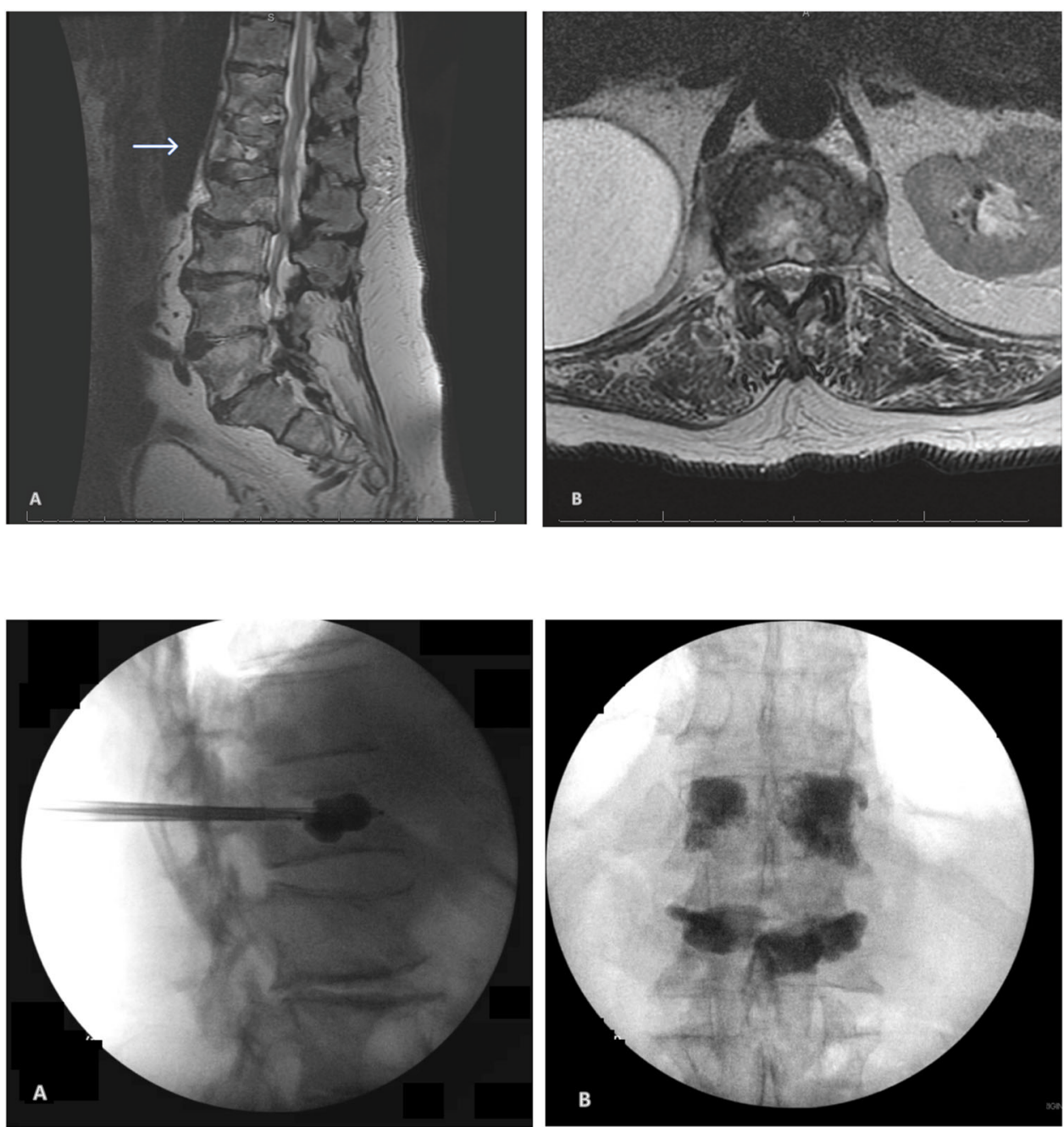

Fig. 2 Intraoperative fluoroscopy of lateral (a) and anteroposterior (b) views of the thoracolumbar spine showing the injection of polymethylmethacrylate following the kyphoplasty of L1 and vertebroplasty of $\mathrm{T} 12$ 


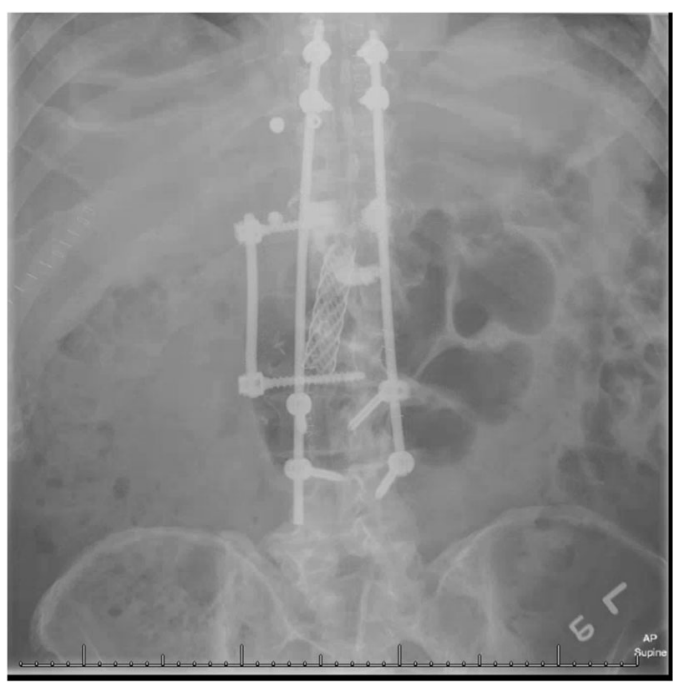

Fig. 3 Upright anteroposterior radiograph of the thoracolumbar spine following an anterior corpectomy from T12-L2 and placement of an intervertebral biomechanical implant and stabilization with posterior T9-L4 instrumentation and fusion

likely secondary to tigecycline. Due to ongoing adverse treatment effects and poor quality of life, the patient and family elected to withdraw aggressive care and continue with palliative measures only.

\section{Discussion}

The identification and diagnosis of VO presents many challenges to clinicians. It is an indolent and slowly progressing disease, often accompanied by nonspecific symptoms, such as back pain, malaise, and fatigue [14]. Although vertebral osteomyelitis is rare with an incidence of $2-4 \%$ of all cases of bone infections, it is a common public health concern in many developing countries. The most common microorganism associated with VO is Staphylococcus aureus. Other organisms implicated include streptococci species, enterobacteriaceae, and enterococci. In developing countries, tuberculosis is the most common cause of $\mathrm{VO}$ presenting with rapid destruction of vertebral bodies and early sparing of disc space [15].

A Pubmed review of the literature published between 1965 and 2017 demonstrated only eight cases of vertebral osteomyelitis caused by $M$. abscessus, half of which occurred in immunocompromised patients. Among the four immunocompromised patients, one received long-term steroid therapy for systemic lupus erythematous, while two had a history of intravenous (IV) drug abuse; one of which was diagnosed with Hepatitis C virus [11, 16, 17]. The final patient had undergone organ transplantation necessitating immunosuppressive therapy [18]. Among the four immunocompetent patients, two cases involved blunt trauma [15]. Only two cases in our literature search revealed an immunocompetent patient without any prior history of trauma or IV drug abuse; one of which resided in a TBendemic region $[19,20]$.

Early diagnosis requires a thorough history, physical exam, laboratory testing, and imaging. Even in disseminated disease, blood cultures are often negative and offer limited value. Definitive diagnosis depends on bone and soft tissue sampling for culture and histologic examination [21]. Nontuberculous mycobacteria, including $M$. abscessus, are naturally occurring organisms found in water and soil and can inhabit body surfaces and secretions through the environment. In most immunocompetent individuals, exposure does not lead to disease. Therefore, isolated cultures of NTM can be often be considered colonizers or contaminants [20].

Recognition of the disease requires a high index of suspicion and an extensive workup. Prompt diagnosis ensures improved long-term outcomes, especially when the clinical course presents as an indolent, prolonged, and destructive process that is unresponsive to empirical antibacterial agents.

Our patient hailed from a non-endemic region and presented with nonspecific symptoms of back pain and a remote history of blunt trauma. Thus there was a low index of suspicion of osteomyelitis as the underlying cause. He continued to have pain while undergoing conservative treatment, prompting operative management and a bone biopsy. A second positive culture via image-guided bone biopsy redemonstrated $M$. abscessus, prompting initiation a multi-drug antibiotic regimen.

As in the majority of cases of vertebral osteomyelitis, initial treatment consists of medical and antibiotic therapy, in conjunction with external bracing. However, $M$. abscessus localized to the spine presents a unique challenge, as there is a lack of consensus on antimicrobial agents, combination therapies, and duration of treatment [22]. Case reports of $M$. abscessus are most commonly related to pulmonary or skin and soft tissue infections, with limited evidence of successful treatment in the spine. Moreover, $M$. abscessus has demonstrated resistance to standard antituberculous agents and antimicrobial therapies [10,22].

The macrolide antibiotic clarithromycin is a mainstay of multi-agent therapy, it is often administered in combination with amikacin, cefoxitin, and tigecycline, but may also be delivered alone. Regimens often consist of weeks to months of parenteral treatment followed by oral therapy. However, certain isolates of $M$. abscessus are not susceptible to macrolide-based chemotherapy. Recent advances have been made possible by new molecular testing methods. Studies have determined that macrolide resistance is conferred by the erm(41) gene in some $M$. abscessus species, rendering macrolide-based chemotherapy against the organism futile. 
Thus molecular testing may further help guide treatment and predict outcomes [10,22].

Surgery has a limited role in the early treatment of vertebral osteomyelitis. Indications are usually reserved for compression or instability leading to a neurologic deficit, or systemic sepsis with discernable foci [23]. However, the recommended mainstay of treatment for chronic vertebral osteomyelitis is an extensive surgical debridement and culture-directed antibiotic therapy.

At present, there are no standard guidelines for treating M. abscessus in the spine. While few prior cases have demonstrated successful outcomes with medical management alone [15, 17, 18, 24], other reports emphasize the necessity of adjunctive surgical management with appropriate antimicrobial therapy [11, 16, 19, 20]. Our study underscores the need for prompt identification and judicious antibiotic administration for $M$. abscessus vertebral osteomyelitis. However, appropriate patient selection when considering an extensive surgical procedure is also critical to achieving a satisfactory clinical outcome.

\section{Compliance with ethical standards}

Conflict of interest The authors have not received grant support nor research funding and do not have any proprietary interests in the materials described in the article.

Publisher's note: Springer Nature remains neutral with regard to jurisdictional claims in published maps and institutional affiliations.

Open Access This article is licensed under a Creative Commons Attribution 4.0 International License, which permits use, sharing, adaptation, distribution and reproduction in any medium or format, as long as you give appropriate credit to the original author(s) and the source, provide a link to the Creative Commons license, and indicate if changes were made. The images or other third party material in this article are included in the article's Creative Commons license, unless indicated otherwise in a credit line to the material. If material is not included in the article's Creative Commons license and your intended use is not permitted by statutory regulation or exceeds the permitted use, you will need to obtain permission directly from the copyright holder. To view a copy of this license, visit http://creativecommons. org/licenses/by/4.0/.

\section{References}

1. Courjon J, Lemaignen A, Ghout I, Therby A, Belmatoug N, Dinh A, et al. Pyogenic vertebral osteomyelitis of the elderly: Characteristics and outcomes. PloS ONE. 2017;12:e0188470.

2. Wilensky AO. Osteomyelitis of the vertebrae. Ann Surg. 1929;89:731.

3. Carragee EJ. Pyogenic vertebral osteomyelitis. JBJS. 1997;79:874-80.

4. Poyanli A, Poyanli O, Akan K, Sencer S. Pneumococcal vertebral osteomyelitis: a unique case with atypical clinical course. Spine. 2001;26:2397.

5. Pruitt TC, Hughes LO, Blasier RD, McCarthy RE, Glasier CM, Roloson GJ. Atypical mycobacterial vertebral osteomyelitis in a steroid-dependent adolescent. A Case Report Spine. 1993;18:2553-5.

6. Jerome M, Slim J, Sison R, Marton R. A case of aerococcus urinae vertebral osteomyelitis. J Glob Infect Dis. 2015;7:85.

7. Nahass RG, Wisneski R, Herman DJ, Hirsh E, Goldblatt K. Vertebral osteomyelitis due to Roseomonas species: case report and review of the evaluation of vertebral osteomyelitis. Clin Infect Dis. 1995;21:1474-6.

8. Lorenz ML, Bouton TC, Caliendo AM. First reported case of vertebral osteomyelitis due to Erysipelothrix rhusiopathiae. ID Cases. 2018;11:3-5.

9. Corrah T, Enoch D, Aliyu S, Lever A. Bacteraemia and subsequent vertebral osteomyelitis: a retrospective review of 125 patients. QJM: an international. J Med. 2010;104:201-7.

10. Petrini B. Mycobacterium abscessus: an emerging rapid-growing potential pathogen. APMIS. 2006;114:319-28.

11. Sarria JC, Chutkan NB, Figueroa JE, Hull A. Atypical mycobacterial vertebral osteomyelitis: case report and review. Clinical infectious diseases. 1998;1:503-5.

12. Petitjean G, Fluckiger U, Schären S, Laifer G. Vertebral osteomyelitis caused by non-tuberculous mycobacteria. Clin Microbiol Infect. 2004;10:951-3.

13. Gopinath K, Singh S. Non-tuberculous mycobacteria in TBendemic countries: are we neglecting the danger? PLoS Negl Trop Dis. 2010;4:e615.

14. Ambrose GB, Alpert M, Neer CS. Vertebral osteomyelitis: a diagnostic problem. JAMA. 1966;197:619-22.

15. Chan ED, Kong P-M, Fennelly K, Dwyer AP, Iseman MD. Vertebral osteomyelitis due to infection with nontuberculous Mycobacterium species after blunt trauma to the back: 3 examples of the principle of locus minoris resistentiae. Clin Infect Dis. 2001;32:1506-10.

16. Maxson S, Schutze GE, Jacobs RF. Mycobacterium abscessus osteomyelitis: treatment with clarithromycin. Infect Dis Clin Pract. 1994;3:203-6.

17. Garcia DC, Sandoval-Sus J, Razzaq K, Young L. Vertebral osteomyelitis caused by Mycobacterium abscessus. BMJ case reports. 2013;2013:bcr2013009597.

18. Silva JT, López-Medrano F, Fernández-Ruiz M, San-Juan R, Ruiz-Cano MJ, Delgado JF, et al. Mycobacterium abscessus pulmonary infection complicated with vertebral osteomyelitis in a heart transplant recipient: case report and literature review. Transplant Infectious Disease. 2015;17:418-23.

19. Kato S, Murakami H, Demura S, Yoshioka K, Hayashi H, Yokogawa N, et al. Vertebral osteomyelitis caused by Mycobacterium abscessus surgically treated using antibacterial iodinesupported instrumentation. Case reports in orthopedics. 2014;2014. https://doi.org/10.1155/2014/197061.

20. Je D, Kang CI, Jeong H, Cho YY, Huh K, Peck KR. Vertebral Osteomyelitis caused by Mycobacterium abscessus in an Immunocompetent Patient. Infection \& Chemotherapy. 2012; 44:530-4.

21. Shimizu H, Mizuno Y, Nakamura I, Fukushima S, Endo K, Matsumoto T. Vertebral osteomyelitis caused by non-tuberculous mycobacteria: case reports and review. J Infect Chemother. 2013;19:972-7.

22. Lee M-R, Ko J-C, Liang S-K, Lee S-W, Yen DH-T, Hsueh P-R. Bacteraemia caused by Mycobacterium abscessus subsp. abscessus and M. abscessus subsp. bolletii: clinical features and susceptibilities of the isolates. Int $\mathrm{J}$ Antimicrob Agents. 2014;43:438-41.

23. Cornett CA, Vincent SA, Crow J, Hewlett A. Bacterial spine infections in adults: evaluation and management. JAAOS-J Am Acad Orthop Surg. 2016;24:11-18.

24. Edwards C, Diveronica M, Abel E. Epidural abscess caused by Mycobacterium abscessus. Am J Case Rep. 2012;13:180. 